\title{
Cognitive and Emotional Empathy in Young Adolescents: an fMRI Study
}

\author{
Eun Jin Kim¹, Jung-Woo Son², Seong Kyoung Park ${ }^{3}$, Seungwon Chung ${ }^{1}$, Hei-Rhee Ghim ${ }^{4}$, \\ Seungbok Lee ${ }^{4}$, Sang-Ick Lee ${ }^{2}$, Chul-Jin Shin ${ }^{2}$, Siekyeong Kim², \\ Gawon Ju', Hyemi Park', and Jeonghwan Lee ${ }^{1}$ \\ ${ }^{1}$ Department of Psychiatry, Chungbuk National University Hospital, Cheongju, Korea \\ ${ }^{2}$ Department of Neuropsychiatry, College of Medicine, Chungbuk National University, Cheongju, Korea \\ ${ }^{3}$ Onyourhan Psychiatric Clinic, Cheongju, Korea \\ ${ }^{4}$ Department of Psychology, Chungbuk National University, Cheongju, Korea
}

\begin{abstract}
Objectives: We investigated the differences in cognitive and emotional empathic ability between adolescents and adults, and the differences of the brain activation during cognitive and emotional empathy tasks.

Methods: Adolescents (aged 13-15 years, $n=14$ ) and adults (aged 19-29 years, $n=17$ ) completed a range of empathic ability questionnaires and were scanned functional magnetic resonance imaging (fMRI) during both cognitive and emotional empathy task. Differences in empathic ability and brain activation between the groups were analyzed.

Results: Both cognitive and emotional empathic ability were significantly lower in the adolescent compared to the adult group. Comparing the adolescent to the adult group showed that brain activation was significantly greater in the right transverse temporal gyrus (BA 41), right insula (BA 13), right superior parietal lobule (BA 7), right precentral gyrus (BA 4), and right thalamus whilst performing emotional empathy tasks. No brain regions showed significantly greater activation in the adolescent compared to the adult group while performing cognitive empathy task. In the adolescent group, scores of the Fantasy Subscale in the Interpersonal Reactivity Index, which reflects cognitive empathic ability, negatively correlated with activity of right superior parietal lobule during emotional empathic situations $(\mathrm{r}=-0.739, \mathrm{p}=0.006)$.

Conclusion: These results strongly suggest that adolescents possess lower cognitive and emotional empathic abilities than adults do and require compensatory hyperactivation of the brain regions associated with emotional empathy or embodiment in emotional empathic situation. Compensatory hyperactivation in the emotional empathy-related brain areas among adolescents are likely associated with their lower cognitive empathic ability.
\end{abstract}

Key Words: Adolescent brain; Empathy; Cognitive empathy; Emotional empathy; Functional magnetic resonance imaging.

Received: April 29, 2020 / Revision: May 25, 2020 / Accepted: June 1, 2020

Address for correspondence: Jung-Woo Son, Department of Neuropsychiatry, College of Medicine, Chungbuk National University, 1 Chungdae-ro, Seowon-gu, Cheongju 28644, Korea

Tel: +82-43-269-6187, Fax: +82-43-267-7951, E-mail: mammosss@hanmail.net

\section{INTRODUCTION}

Adolescence is a time of great change in social cognitive abilities. This includes increased self-consciousness, growing complexity and importance of peer relationships, and an improved understanding of others [1]. As such, interest in adolescents' social brain has increased to reveal the progress of social brain development. Several studies have shown that, in adolescents, brain areas involved in social cognition show different structural and functional characteristics to those of adults. Monk et al. [2] reported adolescents relative to adults

This is an Open Access article distributed under the terms of the Creative Commons Attribution Non-Commercial License (https://creativecommons.org/licenses/by-nc/4.0) which permits unrestricted non-commercial use, distribution, and reproduction in any medium, provided the original work is properly cited. showed greater activation of the anterior cingulate cortex and left orbitofrontal cortex during passive viewing of fearful faces relative to neutral faces. In particular, Burnett et al. [3] reported that in socio-affective scenarios requiring mentalization, adolescents show greater activation than adults, in anterior areas including the left medial prefrontal cortex (mPFC). Additionally, adults show greater activation than adolescents in posterior areas including the left temporal pole. However, social cognition is not limited to mentalization; it involves many other abilities.

Empathy is a fundamental component of social cognition [4]. Recently, attempts to understand it have differentiated between cognitive and emotional empathy $[5,6]$. Cognitive empathy is the ability to engage in the process of adopting 
other's perspectives and represent the mental status of others [6]. It involves the theory of mind (TOM), perspectivetaking, and mentalization [6]. Emotional empathy is the ability to experience affective reactions to the observed experiences of others or share a feeling. It involves emotional contagion, motor emotion, and shared pain [6]. The main brain area associated with cognitive empathy is the ventromedial prefrontal cortex (vmPFC). This area is involved in TOM and self-reflection. In addition, the temporoparietal junction (TPJ), the superior temporal sulcus (STS), and the medial temporal lobe may also be involved in cognitive empathy [6,7]. The brain regions reported to be involved in emotional empathy differ. They include the inferior frontal gyrus and inferior parietal lobule (IPL). These areas are known to form part of the mirror neuron system [6,7]. In addition, given that the anterior cingulate cortex and anterior insula are activated during experiences of shared pain, these regions are also thought to be involved in emotional empathy [6].

In a functional magnetic resonance imaging (fMRI) study, Decety and Michalska [7] found that, in subjects that were a range of ages, a gradual shift from medial to lateral portion of vmPFC when observing painful situation. This pattern suggested emotional empathy presented earlier than cognitive empathy. Additionally, a study on adolescents by Kral et al. [8] found that whilst empathic accuracy, measured from the correlation between perceiver's ratings of a target's emotions relative to the target's ratings of their own emotions in the video, was positively correlated with activation in the prefrontal cortex, TPJ, and STS (areas involved in the cognitive empathy network), it negatively correlated with activation in the IPL (part of the emotional empathy network). Furthermore, while studying event related potentials (ERPs) Mella et al. [9] reported that, compared to adults, adolescents showed a faster early automatic response to painful situations and a greater activation of the late cognitive component in response to neutral stimuli. These studies suggest that adolescents' neural response patterns may differ from adults in situations invoking cognitive or emotional empathy. Currently, there remains a considerable lack of research on this topic.

In research, there is a need for tasks that can accurately differentiate between cognitive and emotional empathy to compare brain responses between adults and adolescents. These works could help understanding how two dimensions of empathy develops through adolescence. Furthermore, studying fMRI during either task clarify social brain development of adolescent. In this study, we explored the differences in brain activity patterns, demonstrated using measures derived from blood oxygenation level dependent (BOLD) fMRI data, between adolescents and adults when performing these tasks.

\section{METHODS}

\section{Participants}

Participants were recruited through online advertisements and leaflets distributed in Chungcheong-do, Republic of Korea. The participants in the adolescent group were aged 1315 years with no history of physical or psychiatric disease. All included adolescents scored below the threshold for disorders in the Kiddie-Schedule for Affective Disorders and Schizophrenia-Present and Lifetime version-Korean version (K-SADS-PL-K) assessment [10]. The adult group participants were aged 19-29 years with no history of physical or psychiatric disease. All included adults scored below the threshold for disorders in the Mini-International Neuropsychiatric Interview (MINI) 6.6.0 [11]. All participants were right-handed. Individuals with an IQ $<70$ and those who could not undergo magnetic resonance imaging (MRI) (e.g., individuals with claustrophobia) were excluded. Participants requiring vision correction wore MRI-compatible glasses during image acquisition to give a corrected visual acuity of 1.0.

This study received ethical approval from the Institutional Review Board of Chungbuk National University (IRB No. CBNU-201701-BM-403-01). Written informed consent was obtained from participants following a thorough explanation of the study objectives and methods. For adolescents, consent was obtained from the participant and their parents whilst for adults, it was obtained from the participant. There were 20 (M: 10, F: 10) and 23 (M: 13, F: 10) consenting participants in the adolescent and adult groups respectively. In the adolescent group, one participant was excluded because results could not be obtained for one of the fMRI tasks and a further five were excluded due to motion artifacts in the fMRI scans. In the adult group, three participants were excluded due to missing questionnaire data, one participant opted out during fMRI acquisition, one participant was excluded due to imaging data error and one participant was excluded due to motion artifacts in the fMRI scans. Resultantly, the final analysis included 14 and 17 individuals in the adolescent and adult groups respectively (Fig. 1).

\section{Assessment methods and instruments}

\section{Intelligence assessment}

The short form of the Korean-Wechsler Intelligence Scale for Children-III (K-WISC-III) test was used to evaluate general intelligence of the adolescent group. It contained the following four subtests: 'Similarities,' 'Arithmetic,' 'Block Design,' and 'Picture Completion.' Scores in these domains are known to correlate positively and strongly with overall intelligence $(\rho=0.85)$ [12]. In the adult group, intelligence was eval- 


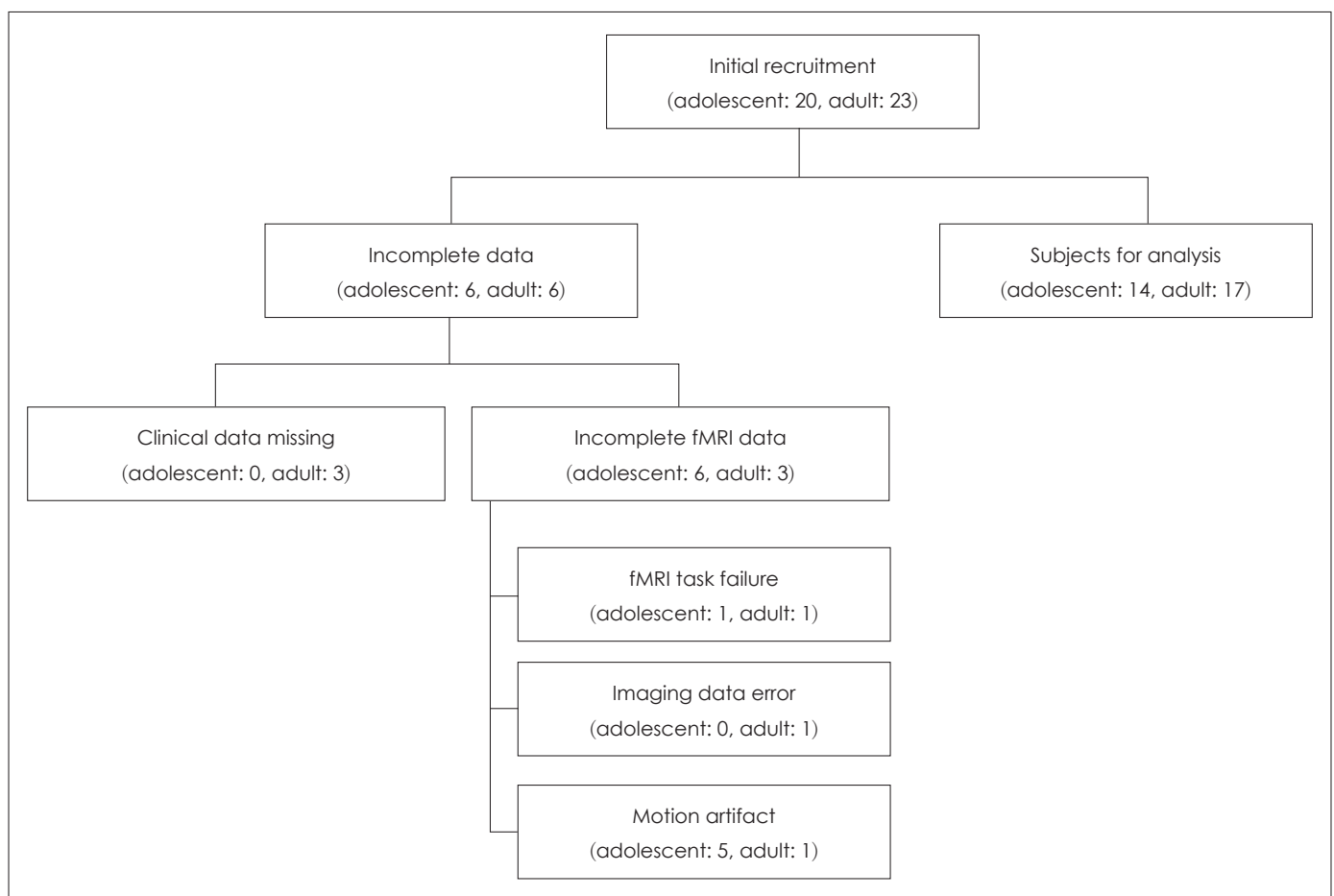

Fig. 1. Study profile.

uated using the short form of the Korean-Wechsler Adult Intelligence Scale-III (K-WAIS-III). The subtests 'Information' and 'Picture Completion' were used to assess general intelligence, as suggested by Kaufman et al. [13]. This method has been reported to have significant sensitivity $(r=0.90)$ and validity $(\mathrm{r}=0.88)$ [13].

\section{Psychiatric evaluation instruments}

The adolescent group was interviewed using the semistructured K-SADS-PL-K to screen for psychiatric illness. It has been reported as reliable and valid for the diagnoses of ADHD, behavioral disorders, tic disorder, depressive disorder, and anxiety disorder [10]. In the adult group, the MINI 6.0.0 interview was used to screen for psychiatric illness. It is based on diagnostic criteria from the ICD-10 and DSM-IV. The Korean version of the MINI has been reported as significantly reliable and valid for mood and anxiety disorders [11].

\section{Empathy-related questionnaires}

The following empathy-related questionnaires were administered to all of the study participants.

\section{Empathy Quotient}

Empathy Quotient (EQ) is a self-reported assessment of an individual's ability and tendencies to understand, make allowances for, and empathize with another's state of mind [14]. The scale consists of 60 questions in total where 40 assess the individual's empathic abilities with the remaining 20 unrelated to empathy. The total score ranges from $0-80$ points with higher scores indicating stronger empathic abilities. The Korean version of the EQ scale shows a reliability coefficient (Cronbach's $\alpha$ ) of 0.79 [15].

\section{Rosenberg Self-Esteem Scale}

The Rosenberg Self-Esteem Scale (RSES) is composed of 10 questions. In the Korean adaptation of this scale, questions are scored on a five-point Likert scale. Higher total scores indicate higher self-esteem. The Cronbach's $\alpha$ of the test is $0.78[16]$.

\section{Interpersonal Reactivity Index}

The Interpersonal Reactivity Index (IRI) is a self-report questionnaire that multidimensionally measures empathic ability [17]. It consists of 4 subscales each containing 7 questions. The subscales are as follows: perspective-taking (IRIPT), which measures individual ability to adopt the perspective of others; fantasy (IRI-FS), which measures the extent to which an individual places themselves in the minds of fictional characters; empathic concern (IRI-EC), which measures the extent to which an individual sympathizes with the concerns of another; and the personal distress (IRI-PD), which measures the discomfort an individual experiences when observing another's distress. In several studies, the IRI-PT and IRI-FS have been associated with cognitive empathic ability, 
with the IRI-EC and IRI-PD related to emotional empathic ability [17-19]. Resultantly, for the purposes of this study, IRIPT and IRI-FS scores were used as measures of cognitive empathic ability whilst IRI-EC and IRI-PD scores were used as measures of emotional empathic ability. Each question is scored on a Likert scale from 0 to 4 points and the total score ranges from 0-112 points. Higher scores indicates higher empathic ability. The Korean version of the IRI has been reported to be significantly valid and reliable [20]. In the present study, the total IRI score (IRI-total) and the IRI-PT, IRI-FS, IRIEC, and IRI-PD subscale scores were all used in analyses.

\section{fMRI scanning and data analysis}

\section{Task stimuli and design during fMRI}

The stimuli used in this study were created by Chung et al. [21] based on the research of Dziobek et al. [19]. They were constructed using E-prime 2.0 (Psychology Software Tools, Sharpsburg, PA, USA) in a block-based design.

The stimuli consisted of 24 photographs of a person against a background situation showing a negative emotional state such as sadness or fear. Photographs were acquired from the International Affective Picture System (IAPS) and other websites. Four blocks each of the cognitive empathy and the emotional empathy tasks were performed using the same photographs. Each block consisted of a $4 \mathrm{~s}$ prompt message and six trials, each of which lasted for $4 \mathrm{~s}$. Between blocks the participants rested for $16 \mathrm{~s}$. The order of the blocks was randomized. The total fMRI scanning time, including $6 \mathrm{~s}$ of dummy scanning, was $5 \mathrm{~min} 58 \mathrm{~s}$. All participants received an explanation of the experimental process and performed one practice round before fMRI scanning. The photographs used in the practice round were different from those used in the fMRI tasks. The tasks and resting blocks are described below.

\section{Cognitive empathy task}

During the cognitive empathy (CE) task, the question "What is the person feeling?" was presented as the informational stimulus, followed by a photograph stimulus. Two different words describing an emotional state were displayed in the bottom left and right of the stimulus. Correct answers were randomized in respect to side. Participants were instructed to press the keypad in the hand corresponding to the word they believed to be correct.

\section{Emotional empathy task}

During the emotional empathy (EE) task, the question "How much are you feeling for the person?" was presented as the informational stimulus, followed by a photograph stimulus. At the bottom left and right of the stimulus, the terms "Less" and "More" were presented respectively. Irrespective of the type of emotion portrayed in the photograph, participants were instructed to press.

\section{Resting block}

In the resting block (R), a "+" sign was displayed in the center of the screen with no other stimulus. Before fMRI scanning, the participant was instructed to focus their gaze and thoughts on the screen when the " + " sign appeared.

\section{fMRI scanning}

Brain images were acquired on a 3.0-tesla Achieva MRI scanner (Philips Medical Systems, Best, the Netherlands) at the Ochang Center of the Korea Basic Science Institute (KBSI). Initially, T1-weighted anatomical gradient echo scan images were acquired. Thereafter functional data during the empathy tasks were collected using an echo planar imaging sequence to collect BOLD data. Slice thickness was $4 \mathrm{~mm}$ and there were no gaps between slices. The MR parameters were as follows: repetition time $(\mathrm{TR})=2000 \mathrm{~ms}$, echo time $(\mathrm{TE})=$ $28 \mathrm{~ms}$, flip angle $=80^{\circ}$, field of view $=240 \times 240 \mathrm{~mm}$, and matrix $=256 \times 256$. For acquisition of T1-weighted images, the slice thickness was $1 \mathrm{~mm}$ and there were no gaps between slices. The MR parameters were as follows: $\mathrm{TR}=6.7 \mathrm{~ms}, \mathrm{TE}=3.2$ $\mathrm{ms}$, flip angle $=9^{\circ}$, field of view $=256 \times 240 \mathrm{~mm}$ and matrix $=$ $256 \times 240$.

\section{fMRI data analysis}

The brain imaging data were analyzed using MATLAB version R2017a (MathWorks, Natick, MA, USA) and SPM8 (Welcome Department of Cognitive Neurology, London, UK). All brain imaging data were motion corrected (realignment), normalized to ascertain the anatomical positions (normalization), and smoothened. To reduce motion artifacts, scans with vertical translations exceeding $1.5 \mathrm{~mm}$ or rotations exceeding $1.5^{\circ}$ were excluded from the analysis. Analysis was performed on the whole brain.

Four contrasts were used in the analysis: cognitive empathy task condition-resting state condition (CE-R), emotional empathy task condition-resting state condition (EE-R), cognitive empathy task condition-emotional empathy task condition (CE-EE), and emotional empathy task conditioncognitive empathy task condition (EE-CE).

Initially, a first-level analysis was conducted for each participant on an individual level followed by a within-group analysis to investigate significantly activated brain regions in the four contrast conditions. Additionally, a between-group analysis was performed to compare differences in the activated brain regions in each contrast condition. In the withingroup analysis, an activated brain region was defined as a 
field of at least 10 voxels with each voxel below the threshold of $\mathrm{p}<0.05$ (FWE corrected). In the between-group analysis, an activated brain region was defined as a field of at least 10 voxels with each voxel below the threshold of $p<0.001$ (uncorrected). To account for sex differences in brain activation patterns in the within-group and between-group analyses, an analysis of covariance was performed with sex as the covariate. In addition, brain regions showing differential activity in the between-group analysis were designated regions of interest (ROIs). The extent of activation within a $5 \mathrm{~mm}$ radius of the most strongly activated voxel was extracted using the SPM-based MarsBaR toolbox (http://marsbar.sourceforge. net), to investigate the level of activation for each condition in each participant. A partial correlation analysis controlling for sex and intelligence was performed, to investigate how brain activity in each ROI correlated with EQ, RSES, and IRI scores.

\section{Statistical analysis}

The sex ratio in the two groups was compared using a chisquare test. Age, IQ, questionnaire results, fMRI task performance, and fMRI response times were tested for normality using the Shapiro-Wilk test. Variables that met the assumptions for normality were compared using independent samples t-tests whilst variables that did not satisfy the conditions for normality were compared using Mann-Whitney U tests. Statistical significance was defined as $\mathrm{p}<0.05$. SPSS Statistics 21 (IBM Corp., Armonk, NY, USA) was used to conduct the statistical analysis.

\section{RESULTS}

\section{Differences between participants in demographic} characteristics and empathic ability

Comparing the adolescent and adult group revealed a sig- nificant difference in age but not in sex or IQ. The adolescent group had significantly lower IRI-total $(\mathrm{t}=-2.067, \mathrm{p}=0.048)$, IRI-PT ( $\mathrm{t}=-3.309, \mathrm{p}=0.003)$, and IRI-EC $(\mathrm{t}=-2.119, \mathrm{p}=0.043)$ scores compared to the adult group. There were no significant differences between the two groups in EQ, RSES, IRIFS, or IRI-PD scores (Table 1).

\section{fMRI task performance and response times}

In the cognitive empathy task, accuracy was significantly lower in the adolescent $(84.82 \pm 7.60 \%)$ compared to the adult group (89.95 $\pm 3.91 \%$; $\mathrm{t}=-2.426, \mathrm{p}=0.022)$ but response times showed no significant difference (adolescent group: 1450.38 $\pm 215.50 \mathrm{~ms}$; adult group: $1500.08 \pm 237.93 \mathrm{~ms} ; \mathrm{t}=-0.604, \mathrm{p}=$ 0.551). In the emotional empathy task, there was no significant difference in the percentage of "More" responses between the adolescent group (56.25 $\pm 17.96 \%)$ and the adult group (65.44 $\pm 17.66 \%$; $\mathrm{t}=-1.431, \mathrm{p}=0.163)$, however, response time was significantly faster in the adolescent group (1321.27士 $250.64 \mathrm{~ms}$ ) compared to the adult group (1614.79 $\pm 301.15 \mathrm{~ms}$; $\mathrm{t}=-2.908, \mathrm{p}=0.007)$.

\section{fMRI results}

\section{The results of within-group analysis}

\section{Adolescent group}

Cerebral regions that showed significant activation in the adolescent group in the CE-R condition were the left lingual gyrus [Brodmann Area (BA) 17], right precentral gyrus (BA $6 / 44$ ), right claustrum, left middle frontal gyrus (BA 46), left inferior frontal gyrus (BA 9), left precentral gyrus (BA 6), right superior frontal gyrus (BA 6), left medial frontal gyrus (BA 8), right precuneus (BA 7), and left inferior parietal lobule (BA 39). Cerebellar regions including the left culmen and right declive also showed significant activation.

Table 1. Demographic and other characteristics of adolescent group and adult group

\begin{tabular}{lcccc}
\hline \multicolumn{1}{c}{ Characteristics } & Adolescent group $(\mathrm{n}=14)$ & Adult group $(\mathrm{n}=17)$ & $\mathrm{t}, \chi^{2}$ or U & $\mathrm{p}$ \\
\hline Male $^{\S}$ & $5(35.71)$ & $10(58.82)$ & $1.642^{11}$ & 0.200 \\
Age $(\mathrm{yr})^{\pi}$ & $14.25 \pm 0.86$ & $23.82 \pm 2.51$ & $238.000^{* *}$ & $<0.001^{\ddagger}$ \\
IQ & $101.86 \pm 7.30$ & $98.82 \pm 6.67$ & $80.500^{* *}$ & 0.128 \\
EQ & $38.79 \pm 11.23$ & $43.53 \pm 9.64$ & -1.266 & 0.216 \\
RSES & $36.64 \pm 3.95$ & $39.12 \pm 5.28$ & -1.450 & 0.158 \\
IRI-total & $68.50 \pm 6.81$ & $74.35 \pm 8.60$ & -2.067 & $0.048^{*}$ \\
IRI-PT & $16.93 \pm 3.15$ & $20.47 \pm 2.81$ & -3.309 & $0.003^{\dagger}$ \\
IRI-FS & $18.14 \pm 2.88$ & $18.35 \pm 4.44$ & -0.152 & 0.880 \\
IRI-EC & $17.36 \pm 2.68$ & $19.47 \pm 2.83$ & -2.119 & $0.043^{*}$ \\
IRI-PD & $16.07 \pm 3.45$ & $16.06 \pm 4.51$ & 0.009 & 0.993 \\
\hline
\end{tabular}

Data are presented as $n(\%)$ or mean \pm standard deviation. ${ }^{*} p<0.05,{ }^{\dagger} p<0.01,{ }^{\ddagger} p<0.001,{ }^{8}$ comparison by chi-square test, " $\chi^{2}$, ${ }^{\pi}$ comparison by Mann-Whitney U test, **Mann-Whitney's U. IQ: intelligence quotient, EQ: empathy quotient, RSES: Rosenberg's Self-Esteem Scale, IRI: Interpersonal Reactivity Index, IRI-total: total score of the IRI, IRI-PT: perspective-taking subscale of the IRI, IRI-FS: fantasy subscale of the IRI, IRI-EC: emotional concern subscale of the IRI, IRI-PD: personal distress subscale of the IRI 
Cerebral regions that showed significant activation in the adolescent group in the EE-R condition were the right inferior frontal gyrus (BA 44), right claustrum, right precentral gyrus (BA 9), right medial frontal gyrus (BA 8), left medial frontal gyrus (BA 6), right superior parietal lobule (BA 7), left middle frontal gyrus (BA 46), left inferior frontal gyrus (BA 45), and right thalamus. In the cerebellum, there was significant activation of the left culmen and the bilateral declives.

In the adolescent group in the CE-EE condition, significant activation was observed in the bilateral lingual gyri (BA 18). No significantly activated brain regions were observed in the adolescent group in the EE-CE condition.

\section{Adult group}

Cerebral regions showing significant activation in the adult group in the CE-R condition were the left lingual gyrus (BA 17), right lingual gyrus (BA 18), bilateral middle frontal gyri (BA 46), right precentral gyrus (BA 9/6), left inferior frontal gyrus (BA 9), left insula (BA 13), right precuneus (BA 7), bilateral superior frontal gyri (BA 6), and left cingulate gyrus (BA 32). In the cerebellum, there was significant activation of the left declive.

The cerebral regions showing significant activation in the adult group in the EE-R condition were the left cuneus (BA 17), left middle occipital gyrus (BA 19), left insula, left middle frontal gyrus (BA 46/6), left inferior frontal gyrus (BA 47), right middle frontal gyrus (BA 46), right claustrum, right inferior frontal gyrus (BA 44), left cingulate gyrus (BA 32), and right superior frontal gyrus (BA 6). There was also significant activation in cerebellar regions such as left declive.

The brain regions showing significant activation in the adult group in the CE-EE condition were the left fusiform gyrus (BA 37), right cuneus (BA 18), and, in the cerebellum, the bilateral declives and the left culmen. No significantly activated brain regions were observed in the adult group in the EE-CE condition.

\section{The results of between-group analysis}

In the CE-R condition, no brain regions showed significantly greater activation in the adolescent compared to the adult group. However, in the EE-R condition, the right transverse temporal gyrus (BA 41), right insula (BA 13), right superior parietal lobule (BA 7), right precentral gyrus (BA 4), and right thalamus all showed significantly greater activation in the adolescent compared to the adult group (Table 2, Fig. 2).

No brain regions showed higher activity in the adult compared to the adolescent group in either of the CE-R and EE-R

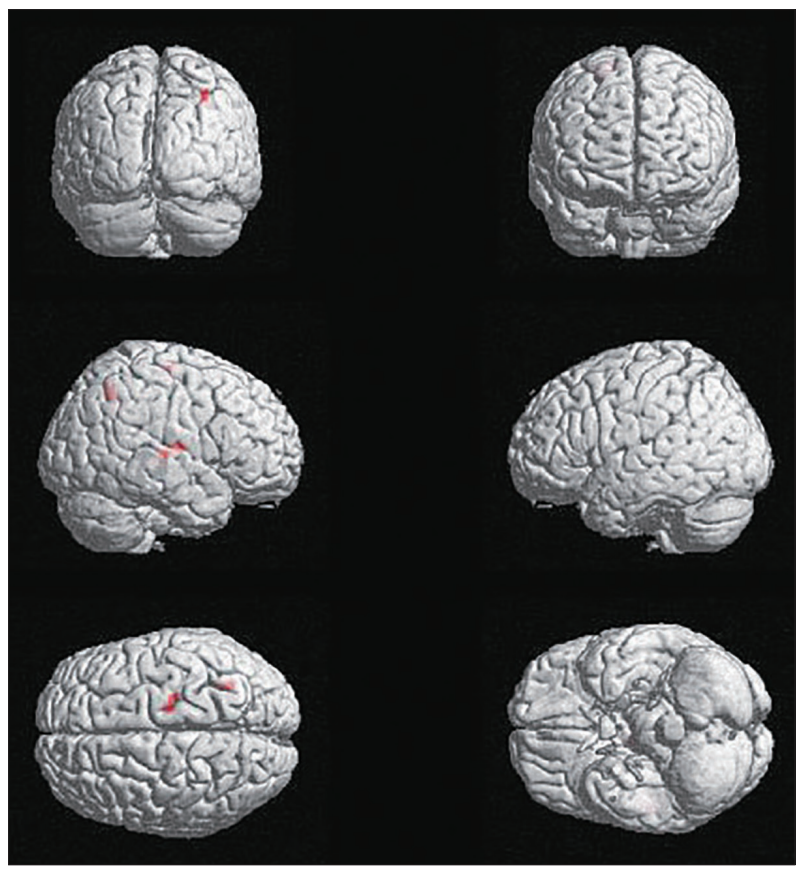

Fig. 2. Significantly activated brain regions of between-group analysis on EE-R contrast (adolescent group > adult group). Right transverse temporal gyrus (BA 41), right insula (BA 13), right superior parietal lobule (BA 7), right precentral gyrus (BA 4), and right thalamus were significantly higher activated. Thresholded at $\mathrm{p}<$ 0.001 uncorrected voxel level, extent threshold 10 voxels. EE: emotional empathy condition, R: resting condition, BA: Brodmann area.

Table 2. The results of between-group analysis when emotional empathy condition compared to resting condition (EE-R contrast)

\begin{tabular}{|c|c|c|c|c|c|c|c|}
\hline \multirow{2}{*}{ Region } & \multirow{2}{*}{ Side } & \multirow{2}{*}{ BA } & \multicolumn{3}{|c|}{$\mathrm{MNI}$ coordinates } & \multirow{2}{*}{$\begin{array}{l}\text { Peak } \\
\text { t-value }\end{array}$} & \multirow{2}{*}{$\begin{array}{c}\text { Cluster size } \\
\text { (voxels) }\end{array}$} \\
\hline & & & $x$ & y & $\mathrm{z}$ & & \\
\hline \multicolumn{8}{|l|}{ Adolescent group > Adult group } \\
\hline Transverse temporal gyrus & Right & 41 & 51 & -22 & 6 & 4.01 & 17 \\
\hline Insula & Right & 13 & 45 & -10 & 10 & 3.90 & - \\
\hline Superior parietal lobule & Right & 7 & 30 & -58 & 46 & 3.98 & 12 \\
\hline Precentral gyrus & Right & 4 & 18 & -19 & 66 & 3.92 & 11 \\
\hline Thalamus & Right & - & 9 & -16 & 14 & 3.87 & 17 \\
\hline Thalamus & Right & - & 6 & -4 & 18 & 3.85 & - \\
\hline \multicolumn{8}{|l|}{ Adult group > Adolescent group } \\
\hline
\end{tabular}

Thresholded at $\mathrm{p}<0.001$ uncorrected voxel level, extent threshold 10 voxels. EE: emotional empathy condition, R: resting condition, BA: Brodmann area, MNI: Montreal Neuroimaing Institute 
Table 3. The results of between-group analysis when cognitive empathy condition compared to emotional empathy condition (CEEE contrast)

\begin{tabular}{|c|c|c|c|c|c|c|c|}
\hline \multirow{2}{*}{ Region } & \multirow{2}{*}{ Side } & \multirow{2}{*}{$\mathrm{BA}$} & \multicolumn{3}{|c|}{ MNI coordinates } & \multirow{2}{*}{$\begin{array}{c}\text { Peak } \\
\text { t-value }\end{array}$} & \multirow{2}{*}{$\begin{array}{c}\text { Cluster size } \\
\text { (voxels) }\end{array}$} \\
\hline & & & $x$ & y & z & & \\
\hline \multicolumn{8}{|l|}{ Adult group>Adolescent group } \\
\hline Superior temporal gyrus & Right & 41 & 42 & -40 & 14 & 4.44 & 12 \\
\hline $\begin{array}{l}\text { Adolescent group > Adult group } \\
\text { (No significant results) }\end{array}$ & & & & & & & \\
\hline
\end{tabular}

Thresholded at $\mathrm{p}<0.001$ uncorrected voxel level, extent threshold 10 voxels. CE: cognitive empathy condition, EE: emotional empathy condition, BA: Brodmann area, MNI: Montreal Neuroimaing Institute
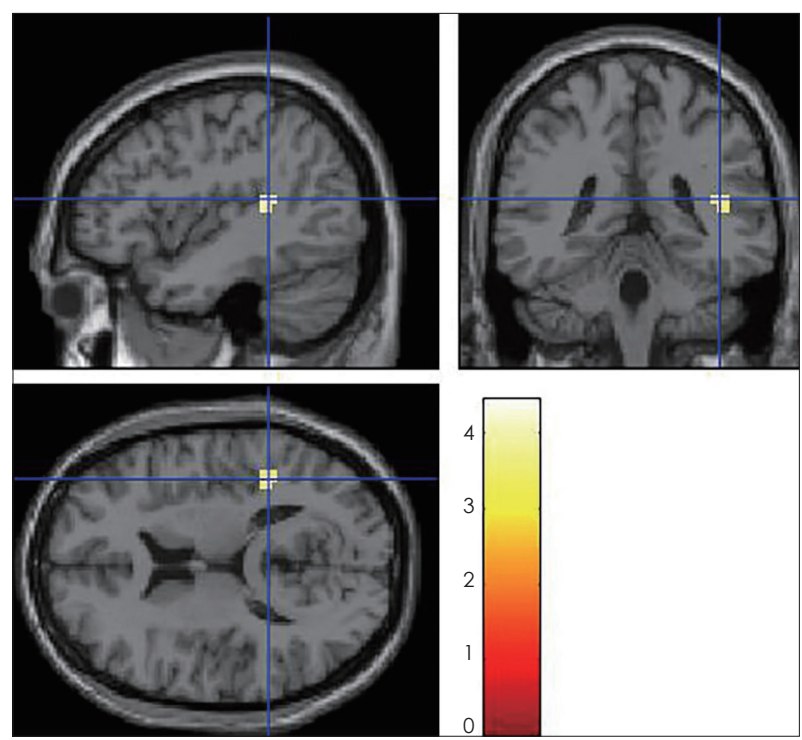

Fig. 3. Significantly activated brain regions of between-group analysis on CE-EE contrast (adult group > adolescent group). Right superior temporal gyrus (BA 41) was significantly higher activated. Thresholded at $\mathrm{p}<0.001$ uncorrected voxel level, extent threshold 10 voxels. CE: cognitive empathy condition, EE: emotional empathy condition, BA: Brodmann area.

conditions. However, in the CE-EE condition, the adult group showed significantly greater activation than the adolescent group in the right superior temporal gyrus (BA 41) (Table 3, Fig. 3).

\section{The results of correlation analysis}

Correlation analysis of the questionnaire and empathy task outcomes with the level of activity in brain regions showing significantly more activation in adolescents than adults in the EE-R condition showed that the level of activity in the right superior parietal lobule (BA 7) showed a significant negative correlation with IRI-FS scores $(\mathrm{r}=-0.739, \mathrm{p}=0.006)$ (Fig. 4). No significant correlations were found between questionnaire and empathy task outcomes analyzed against activity levels in regions showing significantly more activation in adults than adolescents in the CE-EE condition.

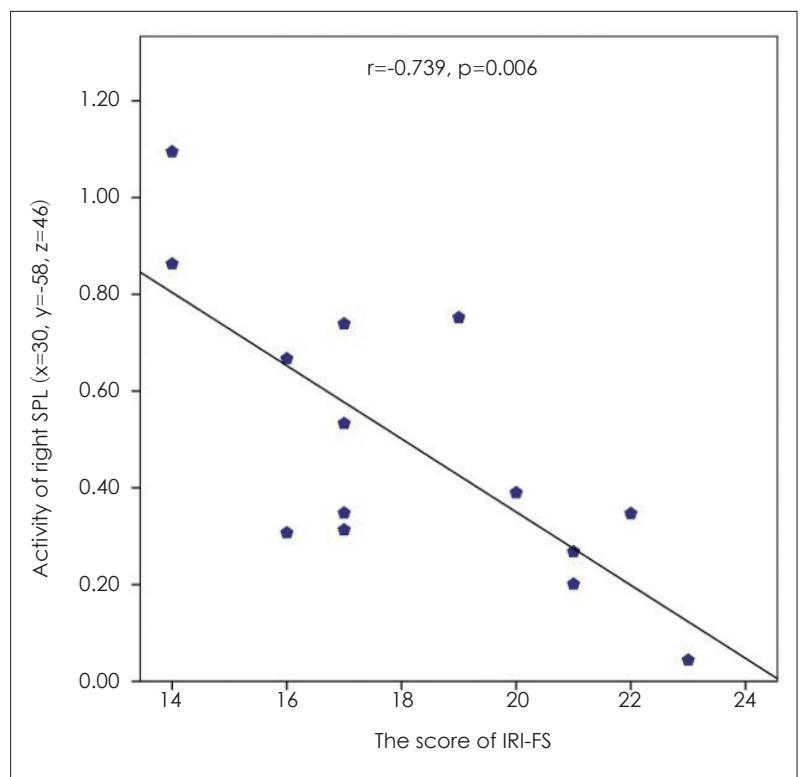

Fig. 4. The results of partial correlation analysis between activity of ROI and scales in EE-R contrast in adolescent group while controlling for the effects of sex and IQ. Negative correlation between right SPL ( $x=30, y=-58, z=46)$ activation (\% signal change) and the score of IRI-FS in EE-R contrast. IRI-FS: fantasy subscale of the Interpersonal Reactivity Index, SPL: superior parietal lobule, $x, y$, and $\mathrm{z}$ : The MNI coordinates $(\mathrm{mm})$ corresponding to the left-right, anterior-posterior, and inferior-superior axes (MNI: Montreal Neurological Institute).

\section{DISCUSSION}

Firstly, it could be questioned whether the participants excluded from the final analysis were homogenous with those who were included. There were no significant differences between the excluded $(n=6)$ and included adolescents $(n=14)$ in age, sex, IQ, or any questionnaire scores. The excluded adults $(n=6)$, only three of which completed the questionnaires, differed significantly in age but not in sex, IQ, or any other questionnaire scores compared to the included adults $(n=17)$. Despite the observed difference in age in the adult group, overall we determined that there was not significant heterogeneity when comparing the included and excluded groups.

Analyzing the empathy characteristics of the participants revealed a significantly lower IRI-total, IRI-PT, and IRI-EC 
scores in the adolescent compared to the adult group. A study by Davis and Franzoi [22] has previously demonstrated that when IRI was measured continually in high school students, IRI-PT and IRI-EC scores significantly increased with increasing age. On the other hand, Hawk et al. [23] have reported that a group of late adolescents (mean age: 18 years) showed significantly higher IRI-PT scores than early adolescents (mean age: 13 years). However, they found no significant difference in IRI-EC scores between the two groups. In the present study, as the two groups were adolescents and adults, it is difficult to make direct comparisons to these previous studies. Nevertheless, our results suggest that adolescents show lower cognitive and emotional empathic ability than adults.

Similarly, in terms of task performance during fMRI, the adolescent group showed lower accuracy than the adult group in the cognitive empathy task. Previously, Greimel et al. [24] found that male adolescents and young male adults showed no differences in a task requiring identification of other's emotions. Contrastingly, Kunzmann et al. [25] found that the ability to accurately recognize another's emotion was significantly worse in a male adolescent group compared to a male adult group. The ability to accurately identify another's emotion is part of emotion recognition [26], which is a component of cognitive empathy $[4,8,19]$. Although results from previous studies are not consistent, our result suggests that adolescents have lower cognitive empathy than adults do.

When performing the emotional empathy task, the adolescent compared to the adult group showed no significant differences in the level of emotional empathy. However, the response times were significantly faster in the adolescent group. Similarly, measured by ERPs, Mella et al. [9] found that an adolescent group showed faster response times when observing painful stimuli than an adult group. Combining these results, it appears that adolescents show faster emotional contagion than adults. It should be noted that this study, like that of Mella et al. [9], used negative emotional stimuli. Resultantly, it can be concluded that adolescents may show rapid emotional empathy followed by an impulsive response to negative emotional stimuli. Furthermore, the reduced accuracy of cognitive empathy discussed above will also affect this impulsive response.

In the within-group analysis, the adolescent and the adult group both showed activation of the precentral gyrus (BA 6), superior frontal gyrus (BA 6), left middle frontal gyrus (BA 46), and left inferior frontal gyrus (BA 9) in the CE-R condition. These regions are also reported to be activated in TOM tasks [27,28]. Furthermore, the adolescent and adult groups both showed activation of the inferior frontal gyrus (BA 44), right claustrum, and middle frontal gyrus (BA 44) in the EE-R condition. The inferior frontal gyrus (BA 44) and middle frontal gyrus (BA 44) are regions that have previously been associated with emotional empathy $[5,6]$. These results show that the fMRI task stimuli in this study appropriately activated brain regions associated with cognitive and emotional empathy.

In the EE-R condition in the between-group analysis, the adolescent group showed significantly more activation than the adult group in the right transverse temporal gyrus (BA 41), right insula (BA 13), right superior parietal lobule (BA 7), right precentral gyrus (BA 4), and right thalamus. These regions are directly and indirectly associated with emotional empathy. Firstly, the transverse temporal gyrus (BA 41) and insula are activated in response to stimuli causing shared pain [29]. Furthermore, the insula is involved in representing current emotional states and is also involved in emotional empathy [30]. Additionally, the thalamus plays an important role in processing emotions [31]; patients with an injury to the thalamus display difficulties recognizing the emotions of others [32]. In particular, the thalamus, as well as the precentral gyrus (BA 4) and the insula (BA 13), are known to be more strongly activated in situations invoking emotional empathy than those invoking cognitive empathy [33]. The superior parietal lobule is reportedly involved in execution of action and in behavior simulation [34]. The precentral gyrus (BA 4), which is the site of the primary motor cortex (M1), activates when imitating another's facial expressions [35]. Thus, activation in the precentral gyrus can be explained in terms of the embodiment of emotional contagion, which is a fundamental element of emotional empathy. Intriguingly, even though the adolescent group showed lower cognitive and emotional empathy when assessed by questionnaires, in the emotional empathy task, they showed greater activation than the adult group in brain regions associated with emotional empathy. These results suggest that adolescents show compensatory hyperactivation of emotional empathy- or embodiment-related brain areas to compensate for their lower emotional empathy ability.

Despite significantly faster reaction times during the emotional empathy task, the adolescent group showed greater activity levels in brain regions related to emotional empathy when compared to the adults. Interpreting this finding requires the consideration of two points. Firstly, during the practice time prior to fMRI acquisition, participants were given sufficient training to maintain their stance from the point of response to each task condition until the resting state block. Secondly, if faster response times mean that participants more easily experience emotional contagion, then the brain regions related to emotional empathy would have remained activated for a longer time. Given these points, it is 
possible that the faster response times could have contributed the hyperactivation of certain brain regions.

Despite lower accuracy in the cognitive empathy task in the adolescent compared to the group, surprisingly, in the CE-R condition, there were no significant differences in neural response in the between-group analysis. Contrastingly, in the CE-EE condition, the adult group showed significantly greater activation than the adolescent group in the posterior part of the superior temporal gyrus (BA 41). The posterior STS is a representative cognitive empathy region $[5,6]$ that is activated in situations involving TOM [36]. This result is consistent with our finding that the adult group showed higher cognitive empathic ability compared to the adolescent group in the questionnaire results. Additionally, it is in concordance with the adult group's higher accuracy in the fMRI cognitive empathy task compared to the adolescent group.

In the correlation analysis, the adolescent group showed a negative correlation between right superior parietal lobule activation during the emotional empathy task and IRI-FS score. The IRI-FS is a subscale that reflects cognitive empathy $[18,19]$. In particular, it measures the extent of emotional involvement with characters appearing in a fictional situation such as a movie or a novel [17]. Adolescents tend to become highly involved in fantasy novels, animations, TV dramas, and/or movies. Interestingly, the results of the present study suggest that, if adolescents show hyperactivation of brain regions involved in emotional empathy when engrossed in a fictional work, their cognitive empathy ability towards the characters may be compromised. In other words, as the emotional empathic brain response increases in adolescents, the ability for cognitive empathy may be impaired. This finding may also indicate that adolescents show less differentiation between cognitive and emotional empathy compared to adults. This will need to be investigated in further studies in lager population with broader age ranges.

This study had the following limitations. Firstly, there was an age gap between the two groups, which omitted individuals 16-18 years old. Therefore, these results cannot explain cognitive and emotional empathic characteristics or functional changes in the brains of these late adolescents. It is possible that the clear age difference between the two groups resulted in distinct differences in the results. Secondly, there were a small number of participants included in the final analysis at 14 and 17 people in the adolescent and adult groups respectively. Were the same analyses to be performed in larger sample groups, more statistically significant results may have been identified. Thirdly, in order to create the empathy tasks, emotional photographs were used from sources such as the IAPS, however, the arousal and valence of each photograph were not normalized during the selection process. The emotional photographs used in this study included emotional behaviors through facial expressions and body language of the individual photographed in addition to the emotional context surrounding them. For this reason, it was difficult to standardize the arousal and valence of each photograph. Fourthly, the fMRI tasks were short. They were designed this way to avoid head movement problems associated with potential reduced task attention in the adolescent group.

\section{CONCLUSION}

This study found that adolescents showed lower cognitive empathy (e.g., perspective-taking) and emotional empathy abilities (e.g., empathic concern) compared to adults. When performing an emotional empathy-related task, relative to the adult group, the brains of adolescents showed compensatory hyperactivation in regions associated with emotional empathy. Adolescents who showed lower cognitive empathy ability (e.g., emotional immersion in fantasy), showed greater activation of relevant brain regions during the emotional empathy task. Hyperactivation of emotional empathy-related brain regions in adolescents may influence their lower cognitive empathy ability. This also suggests that adolescents may show less differentiation between cognitive and emotional empathy mechanisms compared to adults.

\section{Acknowledgments}

Preliminary findings of this study were presented orally at the April 2019 Annual Academic Meeting of the Korean Neuropsychiatric Association.

\section{Conflicts of Interest}

The authors have no potential conflicts of interest to disclose.

\section{Author Contributions}

Conceptualization: Eun Jin Kim, Jung-Woo Son, Seong Kyoung Park, Seungwon Chung, Hei-Rhee Ghim, Seungbok Lee. Data curation: Eun Jin Kim, Jung-Woo Son, Seong Kyoung Park. Formal analysis: Eun Jin Kim, Jung-Woo Son, Seungwon Chung. Funding acquisition: Jung-Woo Son, Seong Kyoung Park. Investigation: Eun Jin Kim. Methodology: Eun Jin Kim, Jung-Woo Son, Seong Kyoung Park, Seungwon Chung, Hei-Rhee Ghim, Seungbok Lee. Project administration: Jung-Woo Son, Hei-Rhee Ghim, Seungbok Lee, Sang-Ick Lee, Chul-Jin Shin, Siekyeong Kim, Gawon Ju, Hyemi Park, Jeonghwan Lee. Resources: Eun Jin Kim, Jung-Woo Son, Seungwon Chung, Sang-Ick Lee, Chul-Jin Shin, Siekyeong Kim, Gawon Ju, Hyemi Park, Jeonghwan Lee. Software: Eun Jin Kim, Jung-Woo Son. Supervision: Sang-Ick Lee, Chul-Jin Shin, Siekyeong Kim, Gawon Ju, Hyemi Park, Jeonghwan Lee. Validation: Jung-Woo Son, Seungwon Chung, Hyemi Park, Jeonghwan Lee. Visualization: Eun Jin Kim. Writing_original draft: Eun Jin Kim. Writing—review \& editing: all authors.

\section{ORCID iDs}

$\begin{array}{ll}\text { Eun Jin Kim } & \text { https://orcid.org/0000-0003-1702-0828 } \\ \text { Jung-Woo Son } & \text { https://orcid.org/0000-0003-4972-3923 } \\ \text { Seong Kyoung Park } & \text { https://orcid.org/0000-0001-9345-9547 }\end{array}$


Seungwon Chung

https://orcid.org/0000-0002-3009-2722

Hei-Rhee Ghim

https://orcid.org/0000-0002-9455-3268

Seungbok Lee

https://orcid.org/0000-0002-3771-7457

Sang-Ick Lee

Chul-Jin Shin

Siekyeong Kim

Gawon Ju

Hyemi Park

https://orcid.org/0000-0001-5134-8064

https://orcid.org/0000-0001-7618-7201

https://orcid.org/0000-0001-8466-9649

https://orcid.org/0000-0002-5358-4578

https://orcid.org/0000-0001-9389-3597

https://orcid.org/0000-0002-9533-848X
Jeonghwan Lee

\section{REFERENCES}

1) Steinberg L, Morris AS. Adolescent development. Annu Rev Psychol 2001;52:83-110.

2) Monk CS, McClure EB, Nelson EE, Zarahn E, Bilder RM, Leibenluft $\mathrm{E}$, et al. Adolescent immaturity in attention-related brain engagement to emotional facial expressions. Neuroimage 2003;20: 420-428.

3) Burnett S, Bird G, Moll J, Frith C, Blakemore SJ. Development during adolescence of the neural processing of social emotion. J Cogn Neurosci 2009;21:1736-1750.

4) Decety J. The neurodevelopment of empathy in humans. Dev Neurosci 2010;32:257-267.

5) Shamay-Tsoory SG, Aharon-Peretz J, Perry D. Two systems for empathy: a double dissociation between emotional and cognitive empathy in inferior frontal gyrus versus ventromedial prefrontal lesions. Brain 2009;132(Pt 3):617-627.

6) Shamay-Tsoory SG. The neural bases for empathy. Neuroscientist 2011;17:18-24.

7) Decety J, Michalska KJ. Neurodevelopmental changes in the circuits underlying empathy and sympathy from childhood to adulthood. Dev Sci 2010;13:886-899.

8) Kral TRA, Solis E, Mumford JA, Schuyler BS, Flook L, Rifken K, et al. Neural correlates of empathic accuracy in adolescence. Soc Cogn Affect Neurosci 2017;12:1701-1710.

9) Mella N, Studer J, Gilet AL, Labouvie-Vief G. Empathy for pain from adolescence through adulthood: an event-related brain potential study. Front Psychol 2012;3:501.

10) Kim YS, Cheon KA, Kim BN, Chang SA, Yoo HJ, Kim JW, et al. The reliability and validity of Kiddie-Schedule for Affective Disorders and Schizophrenia-Present and Lifetime Version-Korean version (K-SADS-PL-K). Yonsei Med J 2004;45:81-89.

11) Yoo SW, Kim YS, Noh JS, Oh KS, Kim CH, Namkoong K, et al. Validity of Korean version of the Mini-International Neuropsychiatric Interview. Anxiety Mood 2006;2:50-55.

12) Jun YS, Hwang ST, Lee SH. Validity of the Korean Wechsler Intelligence Scale for Children-III Short forms. Korean J Clin Psychol 2008;27:277-290.

13) Kaufman AS, Ishikuma T, Kaufman-Packer JL. Amazingly short forms of the WAIS-R. J Psychoeduc Assess 1991;9:4-15.

14) Baron-Cohen $S$, Wheelwright $S$. The empathy quotient: an investigation of adults with Asperger syndrome or high functioning autism, and normal sex differences. J Autism Dev Disord 2004;34:163175.

15) Kim JH, Lee SJ. Reliability and validity of the Korean version of the empathy quotient scale. Psychiatry Investig 2010;7:24-30.

16) Jon BJ. Self-esteem: a test of its measurability. Yonsei Nonchong 1974; 11:107-130

17) Davis MH. Measuring individual differences in empathy: evidence for a multidimensional approach. J Pers Soc Psychol 1983;44:113-
126.

18) Shamay-Tsoory SG, Tomer R, Goldsher D, Berger BD, Aharon-Peretz J. Impairment in cognitive and affective empathy in patients with brain lesions: anatomical and cognitive correlates. J Clin Exp Neuropsychol 2004;26:1113-1127.

19) Dziobek I, Preissler S, Grozdanovic Z, Heuser I, Heekeren HR, Roepke $S$. Neuronal correlates of altered empathy and social cognition in borderline personality disorder. Neuroimage 2011;57:539-548.

20) Kang I, Kee SW, Kim SE, Jeong BS, Hwang JH, Song JE, et al. Reliability and validity of the Korean-version of Interpersonal Reactivity Index. J Korean Neuropsychiatr Assoc 2009;48:352-358.

21) Chung S, Son JW, Lee S, Ghim HR, Lee SI, Shin CJ, et al. Neural correlates of cognitive and emotional empathy in patients with autism spectrum disorder. J Korean Acad Child Adolesc Psychiatry 2016; 27:196-206.

22) Davis MH, Franzoi SL. Stability and change in adolescent self-consciousness and empathy. J Res Pers 1991;25:70-87.

23) Hawk ST, Keijsers L, Branje SJ, Graaff JV, Wied Md, Meeus W. Examining the Interpersonal Reactivity Index (IRI) among early and late adolescents and their mothers. J Pers Assess 2013;95:96-106.

24) Greimel E, Schulte-Rüther M, Fink GR, Piefke M, Herpertz-Dahlmann B, Konrad K. Development of neural correlates of empathy from childhood to early adulthood: an fMRI study in boys and adult men. J Neural Transm (Vienna) 2010;117:781-791.

25) Kunzmann U, Wieck C, Dietzel C. Empathic accuracy: age differences from adolescence into middle adulthood. Cogn Emot 2018; 32:1611-1624.

26) Lawrence K, Campbell R, Skuse D. Age, gender, and puberty influence the development of facial emotion recognition. Front Psychol 2015;6:761.

27) Moriguchi Y, Ohnishi T, Mori T, Matsuda H, Komaki G. Changes of brain activity in the neural substrates for theory of mind during childhood and adolescence. Psychiatry Clin Neurosci 2007;61:355363.

28) Becchio C, Cavallo A, Begliomini C, Sartori L, Feltrin G, Castiello U. Social grasping: from mirroring to mentalizing. Neuroimage 2012; 61:240-248

29) Preis MA, Kröner-Herwig B, Schmidt-Samoa C, Dechent P, Barke A. Neural correlates of empathy with pain show habituation effects. An fMRI Study. PLoS One 2015;10:e0137056.

30) Singer T, Critchley HD, Preuschoff K. A common role of insula in feelings, empathy and uncertainty. Trends Cogn Sci 2009;13:334340 .

31) Ward LM. The thalamus: gateway to the mind. Wiley Interdiscip Rev Cogn Sci 2013;4:609-622.

32) Cheung CC, Lee TM, Yip JT, King KE, Li LS. The differential effects of thalamus and basal ganglia on facial emotion recognition. Brain Cogn 2006;61:262-268.

33) Nummenmaa L, Hirvonen J, Parkkola R, Hietanen JK. Is emotional contagion special? An fMRI study on neural systems for affective and cognitive empathy. Neuroimage 2008;43:571-580.

34) Grèzes J, Decety J. Functional anatomy of execution, mental simulation, observation, and verb generation of actions: a meta-analysis. Hum Brain Mapp 2001;12:1-19.

35) Carr L, Iacoboni M, Dubeau MC, Mazziotta JC, Lenzi GL. Neural mechanisms of empathy in humans: a relay from neural systems for imitation to limbic areas. Proc Natl Acad Sci U S A 2003;100:54975502.

36) Völlm BA, Taylor AN, Richardson P, Corcoran R, Stirling J, McKie $S$, et al. Neuronal correlates of theory of mind and empathy: a functional magnetic resonance imaging study in a nonverbal task. Neuroimage 2006;29:90-98. 